\title{
Laser speckle contrast imaging of abdominal organs in mouse model
}

Evgenya Seryogina, Mikhail Mezentsev, Gennadii
Piavchenko, Viktor Dremin, Igor Kozlov, et al.

Evgenya Seryogina, Mikhail Mezentsev, Gennadii Piavchenko, Viktor Dremin, Igor Kozlov, Anton Sdobnov, Olga Stelmashchuk, Andrian Mamoshin, Andrey Dunaev, "Laser speckle contrast imaging of abdominal organs in mouse model," Proc. SPIE 11065, Saratov Fall Meeting 2018: Optical and NanoTechnologies for Biology and Medicine, 110650Q (3 June 2019); doi: $10.1117 / 12.2523185$ 


\title{
Laser Speckle Contrast Imaging of Abdominal Organs in Mouse Model
}

\author{
Evgenya Seryogina*a, Mikhail Mezentsev ${ }^{\mathrm{a}}$, Gennadii Piavchenko ${ }^{\mathrm{b}, \mathrm{c}}$, Viktor Dreminn ${ }^{\mathrm{a}, \mathrm{d}}$, Igor Kozlov , $^{\mathrm{a}}$ \\ Anton Sdobnov ${ }^{\mathrm{d}}$, Olga Stelmashchuk ${ }^{\mathrm{a}}$, Andrian Mamoshin ${ }^{\mathrm{a}, \mathrm{e}}$, Andrey Dunaev ${ }^{\mathrm{a}}$ \\ ${ }^{a}$ Research and Development Center of Biomedical Photonics, Orel State University, Orel, Russia \\ ${ }^{\mathrm{b}}$ Center for Preclinical Research J.-s.c. "Retinoids", Moscow, Russia \\ ${ }^{c}$ Histology, Cytology and Embriology Department, I.M. Sechenov First Moscow State Medical \\ University (Sechenov University), Moscow, Russia \\ ${ }^{\mathrm{d}}$ Faculty of Information Technology and Electrical Engineering, University of Oulu, Oulu, Finland \\ ${ }^{\mathrm{e}}$ Orel Regional Clinical Hospital, Orel, Russia
}

\begin{abstract}
Diagnosis and treatment of acute destructive pancreatitis is one of the most urgent problems of abdominal surgery. To obtain additional diagnostic information about the microcirculation state and metabolic processes, various imaging techniques are widely used during surgical interventions. One of the most perspective techniques for in vivo microcirculation assessment is laser speckle-contrast imaging (LSCI), allowing for evaluation of blood perfusion. The experimental results showed the possibility to evaluate the blood perfusion of the mouse pancreas in the simulation of ischemia using the LSCI method.
\end{abstract}

Keywords: speckle contrast, speckle pattern, blood flow, mice pancreas, ischemia

\section{INTRODUCTION}

Acute destructive pancreatitis became the one of the urgent problems of emergency surgery of the abdominal cavity in the last decade due to its high mortality and increased frequency of disease complications. Particularly, mortality of pancreatitis is $20-45 \%$ without differentiation of clinical forms. Moreover, mortality reaches up to $85 \%$ in infected pancreatic necrosis, and $100 \%$ in the fulminant course of the disease ${ }^{1,2}$. One of the main problems faced by surgeons is the need to obtain more diagnostic information during surgery operations. An important approach to obtain information about the clinical condition of the patient is the use of informative rapid diagnostic methods allowing the surgeon to timely assess the patient's condition during surgery and choose the right tactics.

The main factors that play a role in the progression of the disease combine microcirculation disorders of the pancreas, ischemic reperfusion injury, the transition from apical apoptosis to necrosis and the complex interaction of these factors. Ischemic-reperfusion injury of the pancreas is one of the trigger mechanisms of its tissue pathomorphosis. These mechanisms had identified subsequent events and manifestations of diseases ${ }^{3,4}$. Violations of microhemodynamics play a leading pathogenetic role in the progression of acute pancreatitis ${ }^{4,5}$.

Currently, a large amount of optical imaging techniques has been implemented for detection of changes in microcirculation in tissues as well as for studying of various biochemical processes closely related to blood supply disorders. At the moment, the most effective methods for determining the basic parameters of microcirculation are the methods of diffuse reflectance imaging ${ }^{6,7}$, laser Doppler imaging ${ }^{8-10}$ and laser speckle-contrast imaging (LSCI) ${ }^{11-14}$.

*e.s.seryogina@gmail.com; phone 74862 419876; bmecenter.ru/en

Saratov Fall Meeting 2018: Optical and Nano-Technologies for Biology and Medicine, edited by

Elina A. Genina, Valery V. Tuchin, Proc. of SPIE Vol. 11065, 110650Q · (c) 2019 SPIE

CCC code: $0277-786 \mathrm{X} / 19 / \$ 18 \cdot$ doi: $10.1117 / 12.2523185$

Proc. of SPIE Vol. 11065110650 Q-1 
LSCI is an effective method for full-scale monitoring of particle dynamics in heterogeneous media. This method is widely used in Biomedicine because it allows researchers and physicians to evaluate the course of physiological processes in vivo with high temporal and spatial resolution. In LSCI, the image of dynamic inhomogeneities is obtained by analyzing the local speckle contrast in the image plane ${ }^{15}$. If inhomogeneous object is illuminated by the coherent light, the randomly changing intensity pattern, produced by random interference in object, can appear. This pattern is widely known as speckle pattern. Movement of particles inside the illuminated medium causes the fluctuations in the intensity of speckle pattern and results in blurred image due to the finite exposure time of detector. Further, temporal and spatial statistics of the speckle pattern can be used for obtaining of information on the motion of scattering particles. The estimation of the contrast of time-averaged dynamic speckles depending on the averaging time of speckle-modulated images forms the data for analysis and processing. The spatial contrast of the speckle image is calculated from a single recorded image of the speckle field in the area.

Analysis of the effectiveness of new diagnostic methods for assessing the degree of acute pancreatitis in humans is impossible without preliminary experimental studies on phantoms and linear animals. There are many methods for modeling pancreatic diseases in the experiment, ranging from mechanical damage to its tissues to complex combined effects. This allows to reproduce acute, chronic and recurrent forms of the disease.

Currently, the most common models of acute pancreatitis ${ }^{2}$ consider the occlusion of the loop of the duodenum, a cholinedeficient diet, supplemented with ethionine, ligation of bile duct and pancreatic duct, injection cerulein, perfusion of the bile duct and pancreatic duct, the introduction of alcohol and the combined perfusion of the bile duct and pancreatic duct with simultaneous cerulein hyperstimulation ${ }^{16-18}$. In our experiment, a model of tissue ischemia was chosen by ligating the vessels of the body of the pancreas to create a blood flow failure in the vessels of the organ.

The aim of the work is to develop a method of application of LSCI for visualization and detection of pathological processes in various organs of the abdominal cavity by means of complex modeling of acute pancreatitis using pancreatic ischemia in mice.

\section{MATERIAL AND METHODS}

The following LSCI-based experimental setup illustrated on the Figure 1 has been used in this study: $10 \mathrm{~mW}$ laser source working at $635 \mathrm{~nm}$ (Edmund Optics Inc, USA) illuminated the object. Further, CMOS- camera DCC 3260M (Thorlabs, USA) with $1936 \times 1216$ pixels and $5.86 \mu \mathrm{m}$ pixel size was used in combination with $34 \mathrm{~mm}$ Mitutoyo Plan Apochromat Objective MY5X-802 (Thorlabs, USA) for raw speckle image acquisition. The obtained images were processed by a custom-developed algorithm in the offline regime using a MATLAB r2017b software environment. The spatial algorithm using $7 \times 7$ sliding window has been used for speckle contrast images obtaining . Totally, 20 speckle contrast images has been calculated and averaged in final speckle contrast image. Thus, the calculation of the final speckle contrast image was carried out according to the Eq. 1:

$$
K=\left\langle\frac{\sigma_{N}}{\langle I\rangle_{N}}\right\rangle_{k},
$$

where \langle\rangle - the symbol of averaging; $N$ - the window of averaging $N \mathrm{x} N(N=7) ; k$ - the number of consecutive frames $(k=20) ;\langle I\rangle_{N}$-average intensity in the window $N \mathrm{x} N ; \sigma_{N}-$ standard deviation in the window $N \mathrm{x} N$.

Prior to conducting animal studies, the flow phantom has been created for setup calibration and testing of its sensitivity. The phantom consisted of a capillary tube (diameter $1 \mathrm{~mm}$ ). 20\% Intralipid suspension was pumped through the capillary tube by a syringe. Changes in the speed of movement of the Intralipid in the tube were recorded by a camera, then, postprocessing of data was performed according to Eq. 1 




Figure 1 - The setup with capillary tube and syringe.

Experimental studies with animal model were performed on clinically healthy male Balb/c mice ( $\mathrm{n}=6$ in the group). Mice were obtained from the FSBI SCBT FMBA of. Russian Federation (“Andreevka" vivarium). Before the animals transfer to the clean area of the vivarium and the beginning of the experiment, the animals were kept in quarantine for 14 days. When placed in quarantine, the veterinarian conducted a primary assessment of the animals condition with the introduction of the examination results in the relevant statement and examined animals daily. The basic rules of maintenance and care corresponded to the standards of the sanitary rules for the arrangement, equipment and maintenance of experimental biological clinics and in the guide "Laboratory animals"19. All procedures for routine animal care were carried out in accordance with the Standart operational procedures of the J.-s.c. "Retinoids".

During the study, mice were anesthetized with Zolilet 100 (Vibrac, France), respectively, at standard dosages. The animal was fixed on a special platform in the position on the back. For each animal, a transverse laparotomy was performed. With the help of the tools, access was made to the upper part on the back wall of the abdominal cavity in the retroperitoneal space. After the opening of the operating field, the complex of organs containing the pancreas was carried out to the laboratory table. The body of the pancreas was isolated and ligature from polyester thread was applied. In addition, a cotton swab soaked in a $0.9 \%$ solution of sodium chloride (normal saline) was placed in the operating field. Then, the animal was placed under the optical system to visualize the study area and record the frame sequences (see Figure 2). The registration of the speckle-contrast image of the tissue was made on the setup before and after 1, 3, 6, 9 and $12 \mathrm{~min}$ the imposition of the ligature.

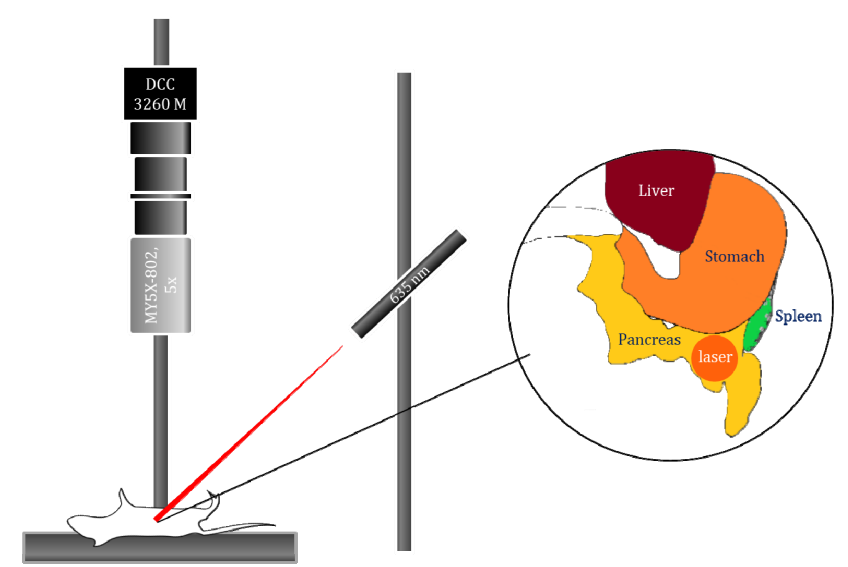

Figure 2 - The setup with the area of laser irradiation of the body of the pancreas. 


\section{EXPERIMENTAL RESULTS AND DISCUSSION}

To check the sensitivity of the installation relatively the change in speed, a sequence of 20 frames was recorded for flow phantom. Three sequences of frames with capillary tube were registered: empty tube, with directed flow of fluid ; with Brownian motion of intralipid. After frames processing, speckle contrast images were obtained (see Figure 3).
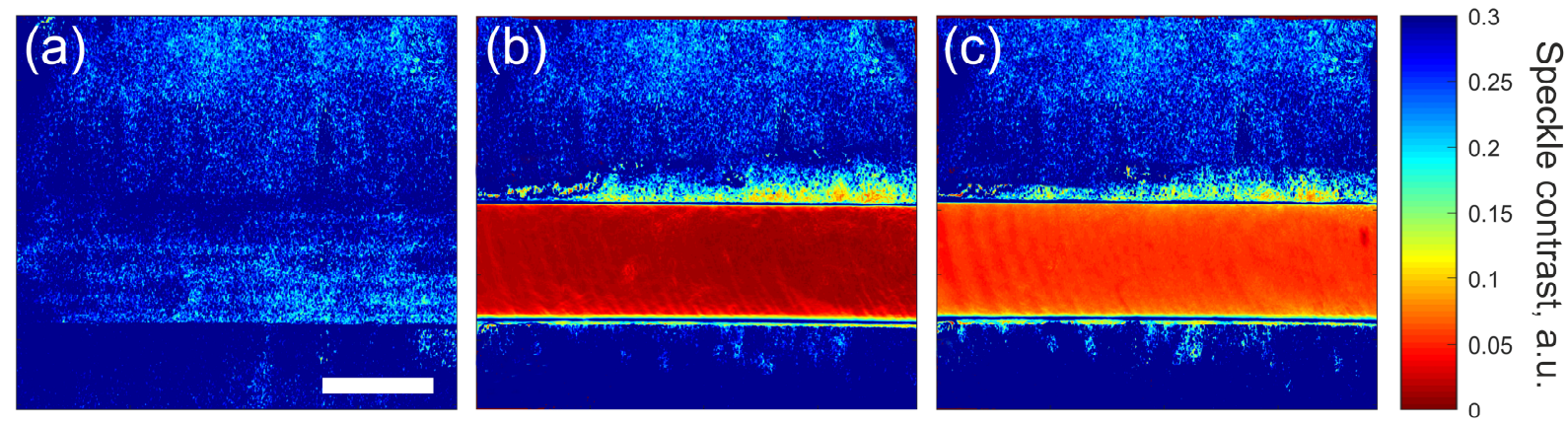

Figure 3 - Speckle contrast images from the phantom ( $\mathrm{a}$ - without intralipid, $\mathrm{b}$ - fluid with direct flow, $\mathrm{c}$ - fluid with Brownian motion). Scale bar equals $1 \mathrm{~mm}$.

Based on the analysis of the obtained frames, it is possible to detect the flow rate of the fluid with the help of the assembled setup. As the result, it was suggested that a similar sensitivity will also be noted for blood flow in a microvasculature.

Then, the developed setup is showed in Figure 3 was used to record the sequence of frames in mice. For each stage, a sequence of 20 frames was recorded. After image processing the space-time algorithm obtained spectral contrast images (see Figure 4).
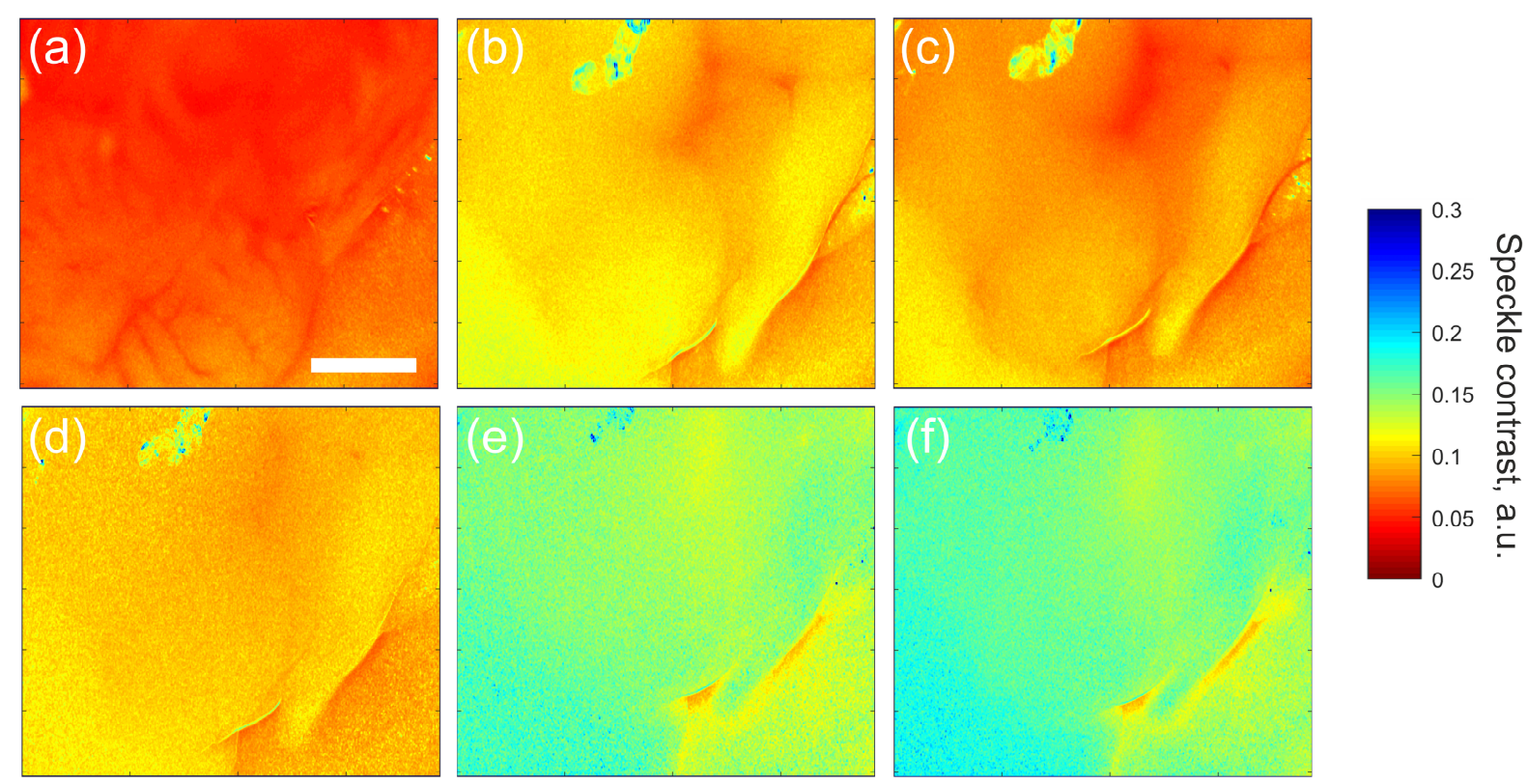

Figure 4 - Speckle images from mice before (a) and 1 (b), 3 (c), 6 (d), 9 (e) and 12 (f) min after ligating pancreas. Scale bar equals $1 \mathrm{~mm}$. 
The average contrast ratio of the speckle image in the area of 100x100 pixels was calculated using the Eq. 1 (see Figure 5).

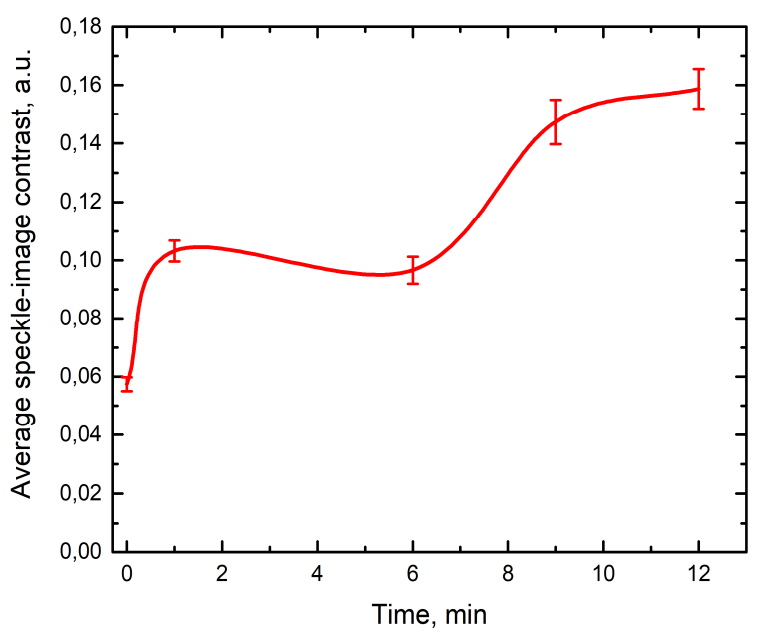

Figure 5 - Evaluation of averaged speckle contrast value taken within part of camera field of view (100 x 100 pixels) during ligation process.

Figure 5 showed ischemia development after ligature registered by speckle-contrast imaging. Increment in averaged speckle contrast value indicates a slowing of blood microcirculation in the pancreas. A decrease in the point corresponding to 6 minutes of the experiment may indicate the occurrence of venous blood flow in the body in the absence of arterial blood flow ${ }^{20}$. This effect is observed with the usual occlusion of the limbs. After 6 minutes, the increasing trend was stayed to the end of recording.

\section{CONCLUSION}

Performed experiments showed differences in the characteristics of the speckle fields of non-ischemic and ischemic tissue. Changes in the microcirculation registered by LSCI allow physicians to assess the degree of development of pathological disorders.

Preliminary data suggest that the registration of changes by laser speckle contrast imaging is capable for representing different degrees of blood perfusion failure of pancreatic tissue in complex modeling of ischemia. The experiment demonstrated a difference in the contrast of spotty fields in healthy and pathologically altered tissue.

The presented method of imaging potentially allows physicians to find out the degree of organ blood supply during the operation. Also, the development can provide additional diagnostic information and allows the doctor to adjust the course of the operation. Despite the fact that there are respiratory and cardiac oscillations of the tissue, it is planned to reduce the influence of these factors with the help of image processing and stabilization algorithms. The main results of this work will be used to develop a laparoscope with an integrated LSCI channel for intraoperative tissue analysis.

\section{ACKNOWLEDGMENTS}

The study was funded by the Russian Science Foundation in according with the research project №18-15-00201. Anton Sdobnov was supported by Finnish Cultural Foundation (00180998) grant. 


\section{REFERENCES}

[1] Al Mofleh, I.-A., "Severe acute pancreatitis: pathogenetic aspects and prognostic factors.," World J. Gastroenterol. 14(5), 675-684 (2008).

[2] Su, K. H., Cuthbertson, C. and Christophi, C., "Review of experimental animal models of acute pancreatitis.," HPB (Oxford). (2006).

[3] Prinz, R. A., "Mechanisms of acute pancreatitis. Vascular etiology.," Int. J. Pancreatol. 9, 31-38 (1991).

[4] Broe, P. J., Zuidema, G. D. and Cameron, J. L., "The role of ischemia in acute pancreatitis: studies with an isolated perfused canine pancreas.," Surgery 91(4), 377-382 (1982).

[5] Cappell, M. S., "Acute pancreatitis: etiology, clinical presentation, diagnosis, and therapy.," Med. Clin. North Am. (2008).

[6] Nishidate, I., Ishizuka, T., Mustari, A., Yoshida, K., Kawauchi, S., Sato, S. and Sato, M., "Evaluation of Cerebral Hemodynamics and Tissue Morphology of In Vivo Rat Brain Using Spectral Diffuse Reflectance Imaging.," Appl. Spectrosc. 71(5), 866-878 (2017).

[7] Potapova, E. V, Dremin, V. V, Zherebtsov, E. A., Makovik, I. N., Zherebtsova, A. I., Dunaev, A. V, Podmasteryev, K. V, Sidorov, V. V, Krupatkin, A. I., Khakhicheva, L. S. and Muradyan, V. F., "Evaluation of microcirculatory disturbances in patients with rheumatic diseases by the method of diffuse reflectance spectroscopy," Hum. Physiol. 43(2), 222-228 (2017).

[8] Essex, T. J. and Byrne, P. O., "A laser Doppler scanner for imaging blood flow in skin.," J. Biomed. Eng. 13(3), 189-194 (1991).

[9] Phillips, A. R., Farrant, G. J., Abu-Zidan, F. M., Cooper, G. J. and Windsor, J. A., "A method using laser Doppler flowmetry to study intestinal and pancreatic perfusion during an acute intestinal ischaemic injury in rats with pancreatitis.," Eur. Surg. Res. 33(5-6), 361-369 (2001).

[10] Dremin, V. V, Zherebtsov, E. A., Makovik, I. N., Kozlov, I. O., Sidorov, V. V, Krupatkin, A. I., Dunaev, A. V, Rafailov, I. E., Litvinova, K. S., Sokolovski, S. G. and Rafailov, E. U., "Laser Doppler flowmetry in blood and lymph monitoring, technical aspects and analysis," Proc. SPIE 10063, 1006303 (2017).

[11] Vilenskii, M. A., Agafonov, D. N., Zimnyakov, D. A., Tuchin, V. V and Zdrazhevskii, R. A., "Specklecorrelation analysis of the microcapillary blood circulation in nail bed," Quantum Electron. 41(4), 324-328 (2011).

[12] Tuchin, V., [Tissue optics : light scattering methods and i nstruments f or medical diagnosis] (2015).

[13] Sdobnov, A., Bykov, A., Molodij, G., Kalchenko, V., Jarvinen, T., Popov, A., Kordas, K. and Meglinski, I., "Speckle dynamics under ergodicity breaking," J. Phys. D. Appl. Phys. 51(15) 155401 (2018).

[14] Sdobnov, A., Bykov, A., Popov, A., Zherebtsov, E. and Meglinski, I., "Investigation of speckle pattern dynamics by laser speckle contrast imaging," Proc. SPIE 10685, 1068509 (2018).

[15] Zakharov, P., Völker, A., Buck, A., Weber, B. and Scheffold, F., "Quantitative modeling of laser speckle imaging," Opt. Lett. 31(23), 3465-3467 (2006).

[16] Mansfield, C., "Pathophysiology of Acute Pancreatitis: Potential Application from Experimental Models and Human Medicine to Dogs," J. Vet. Intern. Med. 26(4), 875-87 (2012).

[17] Klar, E., Rattner, D. W., Compton, C., Stanford, G., Chernow, B. and Warshaw, A. L., "Adverse effect of therapeutic vasoconstrictors in experimental acute pancreatitis.," Ann. Surg. 214(2), 168-174 (1991).

[18] Timoshina, P., Bucharskaya, A., Alexandrov, D. and Tuchin, V., "Study of blood microcirculation of pancreas in rats with alloxan diabetes by Laser Speckle Contrast Imaging," J. Biomed. Photonics Eng. 3, 20301 (2017).

[19] Environment Directorate OECD., "OECD series on principles of good laboratory practice and compliance monitoring," Ann. Ist. Super. Sanita (1997).

[20] Kalchenko, V., Brill, A., Bayewitch, M., Fine, I., Zharov, V., Galanzha, E., Tuchin, V. and Harmelin, A., "In vivo dynamic light scattering imaging of blood coagulation," J. Biomed. Opt. 12(5), 052002 (2007). 